\title{
Miopatías relacionadas a colágeno VI. Cuando sospecharlas, cómo identificarlas. Aporte de la resonancia magnética muscular
}

\author{
Collagen VI related myopathies. When to suspect, how to identify. \\ The contribution of muscle magnetic resonance
}

\author{
Bernardita Suárez ${ }^{a}$, Andrés Lozano-Arango $^{a}$, Diego Araneda ${ }^{b}$, Fanny Cortés $^{c}$, Cecilia Hervias ${ }^{a, d}$, \\ Giancarlo Calcagno $^{\mathrm{a}, \mathrm{d}}$, Ximena Ortega ${ }^{\mathrm{b}, \mathrm{c}}$, Claudia Castiglioni ${ }^{\mathrm{a}}$
}

\author{
aPrograma Enfermedades Neuromusculares y Trastornos Motores, Departamento de Neurología Pediátrica, Clínica Las Condes, Santiago. Chile

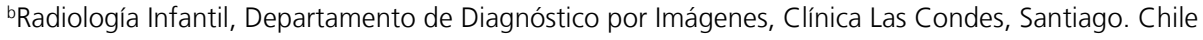 \\ 'Centro de Enfermedades Raras, Clínica las Condes, Santiago. Chile \\ 'Kinesiología, Centro de Medicina del Deporte, Clínica Las Condes, Santiago. Chile
}

Recibido el 27-12-2017; aceptado el 29-03-2018

\begin{abstract}
Resumen
Las miopatías secundarias a mutaciones en el colágeno VI (M-COLVI) son las más frecuentes en el hemisferio norte, afectando población adulta y pediátrica. No existen datos de su prevalencia en Latinoamérica. Se caracterizan por presentar una gran variabilidad clínica, desde fenotipos severos, como la distrofia muscular congénita de Ullrich (DMCU), a intermedios y leves como la Miopatía de Bethlem (MB). Su inicio también es variable y se extiende desde el período de recién nacido hasta la vida adulta. Dada la presencia de hiperlaxitud articular, el diagnóstico diferencial se debe realizar con diversas enfermedades del tejido conectivo. El algoritmo diagnóstico clásico en muchos pacientes ha sido insuficiente para orientar el estudio genético de forma adecuada, y a partir de esto la resonancia magnética muscular ha emergido como una herramienta de gran utilidad para una mejor aproximación diagnóstica de ésta y otras patologías musculares. Esta revisión tiene como objetivo examinar las formas de presentación, características clínicas, estudio diagnóstico específico, diagnóstico diferencial y manejo de una de las patologías musculares herediatarias más frecuentes, con énfasis en el aporte de la resonancia magnética muscular.
\end{abstract}

\section{Abstract}

Myopathies secondary to collagen VI mutations (COLVI-M) are the most frequent in the northern hemisphere, affecting the adult and pediatric population. There are no data on its prevalence in Latin America. They are characterized by a great clinical variability, from severe phenotypes, such as Ullrich congenital muscular dystrophy (UCMD), to intermediate and mild ones such as Bethlem myopathy (BM). Its onset is also variable and extends from the neonatal period to adulthood. Given the pre-
Palabras clave: Colágeno VI; Miopatías congénitas;

Resonancia magnética; Músculo

Keywords: Collagen VI; Congenital Myopathies; Magnetic resonance imagining;

Muscle 
sence of joint hypermobility, the differential diagnosis should be made with various connective tissue diseases. The classical diagnostic algorithm in many patients has been insufficient to guide the genetic study in an adequate way, and from this the muscular magnetic resonance imaging has emerged as a very useful tool for a better diagnostic approach of this and other muscular pathologies. This objective of this review is to study the forms of presentation, clinical characteristics, specific diagnostic study, differential diagnosis and management of one of the most frequent hereditary muscular pathologies, with emphasis on the contribution of muscle magnetic resonance imaging.

\section{Introducción}

Las enfermedades neuromusculares hereditarias abarcan al menos 16 subgrupos de patologías, clasificadas según el lugar de compromiso, desde el músculo, pasando por la unión neuromuscular y los nervios periféricos hasta llegar a las motoneuronas de la asta anterior medular. Particularmente, dentro de las patologías que afectan al músculo destacan 3 de estos subgrupos: las miopatías congénitas, las distrofias musculares congénitas y las distrofias musculares, habiéndose descrito más de 240 fenotipos clínicos para $\operatorname{estas}^{1}$ (Tabla 1). El tipo de herencia es variable e incluye segregaciones de tipo autosómica dominante y recesiva; ligada al cromosoma $\mathrm{X}$ y también mitocondrial. El conocimiento actual de la fisiología de la fibra muscular y de sus diversos componentes estructurales y enzimáticos, han contribuido a la identificación de al menos 67 genes responsables de los fenotipos hasta ahora descritos ${ }^{1,2}$. Estas patologías presentan diverso grado de limitación funcional por debilidad de la musculatura esquelética y contracturas articulares secundarias, pudiendo además asociarse a compromiso cardíaco como miocardiopatías y trastornos de la conducción, y también a compromiso respiratorio con afectación de la mecánica ventilatoria ${ }^{3}$. Respecto a la epidemiología de estas enfermedades, no existen datos exactos debido a las limitaciones en la confirmación diagnóstica con estudios de genética molecular que permitan determinar la alteración subyacente, encontrando en poblaciones representativas, prevalencias de 1:26.000 en EE. UU. para las miopatías congénitas ${ }^{4} \mathrm{e}$ incidencias de 1:16.000 para las distrofias musculares congénitas en Suecia Oriental ${ }^{5}$.

Dentro del grupo de miopatías congénitas, las secundarias a mutaciones del colágeno VI (M-COLVI) representan a su vez un amplio espectro de fenotipos que incluyen un compromiso severo como el de la Distrofia Muscular Congénita de Ullrich (DMCU), a un compromiso más leve como la Miopatía de Bethlem (MB). Si bien los estudios epidemiológicos son escasos, se trata de la miopatía congénita más frecuente en Norte América y la segunda más frecuente en Japón, con prevalencias descritas en el norte de Inglaterra de 0.13 casos por 100.000 habitantes para DMCU y 0,77 casos por 100.000 para la $\mathrm{MB}^{6}$. En Chile y Latinoamérica no existen datos de prevalencia e incidencia de estas enfermedades, existiendo sólo reportes de casos aisla$\operatorname{dos}^{7,8}$. El presente trabajo tiene como objetivo revisar el conocimiento actual de este grupo de patologías, con énfasis en la presentación clínica y particularidades fenotípicas que permiten alertar la sospecha diagnóstica, así como destacar el avance adquirido en los últimos años en los métodos de apoyo diagnóstico, especialmente el aporte de la resonancia magnética muscular, que han permitido acortar la odisea diagnóstica, mejorando el tratamiento y seguimiento de estos pacientes.

\section{Miopatías relacionadas a colágeno VI}

Las M-COLVI son causadas por mutaciones en algunos de los tres genes (COL6A1, COL6A2 y COL6A3) que codifican las cadenas alfa principales del colágeno tipo $\mathrm{VI}^{9,10}$. El colágeno VI es uno de los componentes principales de la matriz extracelular, formando una red microfibrilar estrechamente relacionada con la célula y la membrana basal de múltiples tejidos, incluidos músculos, piel, tendones, cartílagos, discos intervertebrales, lentes, órganos internos y vasos sanguíneos ${ }^{11}$. Fisiopatológicamente, las mutaciones del COL6 generan una disfunción mitocondrial y fenómenos apoptóticos de la fibra muscular, los que se ven amplificados por defectos en los mecanismos de autofagia ${ }^{12}$.

Clínicamente, las M-COLVI son un espectro de enfermedades que comparten en mayor o menor grado una serie de características fenotípicas como la hiperlaxitud y contracturas articulares ${ }^{13}$. Otro órgano comprometido es la piel, con hallazgos que incluyen la hiperqueratosis pilar, presente habitualmente en la superficie extensora de muslos y brazos; cicatrización queloide y en "papel de cigarro"(Figura 2); piel seca o rugosa y piel suave con exceso de pliegues palmares y plantares en pacientes jóvenes con $\mathrm{DMCU}^{8,14}$. Ninguna de las M-COLVI se ha asociado a discapacidad intelectual o trastornos del aprendizaje, así como tampoco hay evidencia de compromiso cardíaco, aún en las formas de mayor severidad ${ }^{15}$.

Podemos distinguir al menos tres fenotipos clínicos principales de M-COLVI: 


\section{Tabla 1. Listado por fenotipo de las principales miopatías congénitas y distrofias musculares congénitas ${ }^{1}$}

\begin{tabular}{lll}
\hline Miopatías congénitas & Distrofias musculares congénitas & $\begin{array}{l}\text { Distrofias musculares congénitas por defecto } \\
\text { de la glicosilación }\end{array}$ \\
\hline Nemalínica & Miopatía de Bethlem & Fukuyama \\
Con desproporción de fibras & De Ullrich & Síndrome de Walker-Warburg \\
Miotúbulos & Relacionada a COL12A1 & Enfermedad musculo-ojo-cerebro \\
Centronuclear & Con déficit de merosina & Con hipoglicosilación del distroglicano \\
Central core & Síndrome de espina rígida & \\
Multiminicore & Por defecto de la lámina AVC & \\
Con cuerpos hialinos & Con defecto de la integrina & \\
Con cuerpos de inclusión & Con defecto de la dinamina 2 & \\
"Cap myopathy" & \\
Con uniformidad de fibras tipo 1 & \\
Sarcotubular & \\
Con cardiomiopatía fatal & \\
Asociadas a miosina &
\end{tabular}

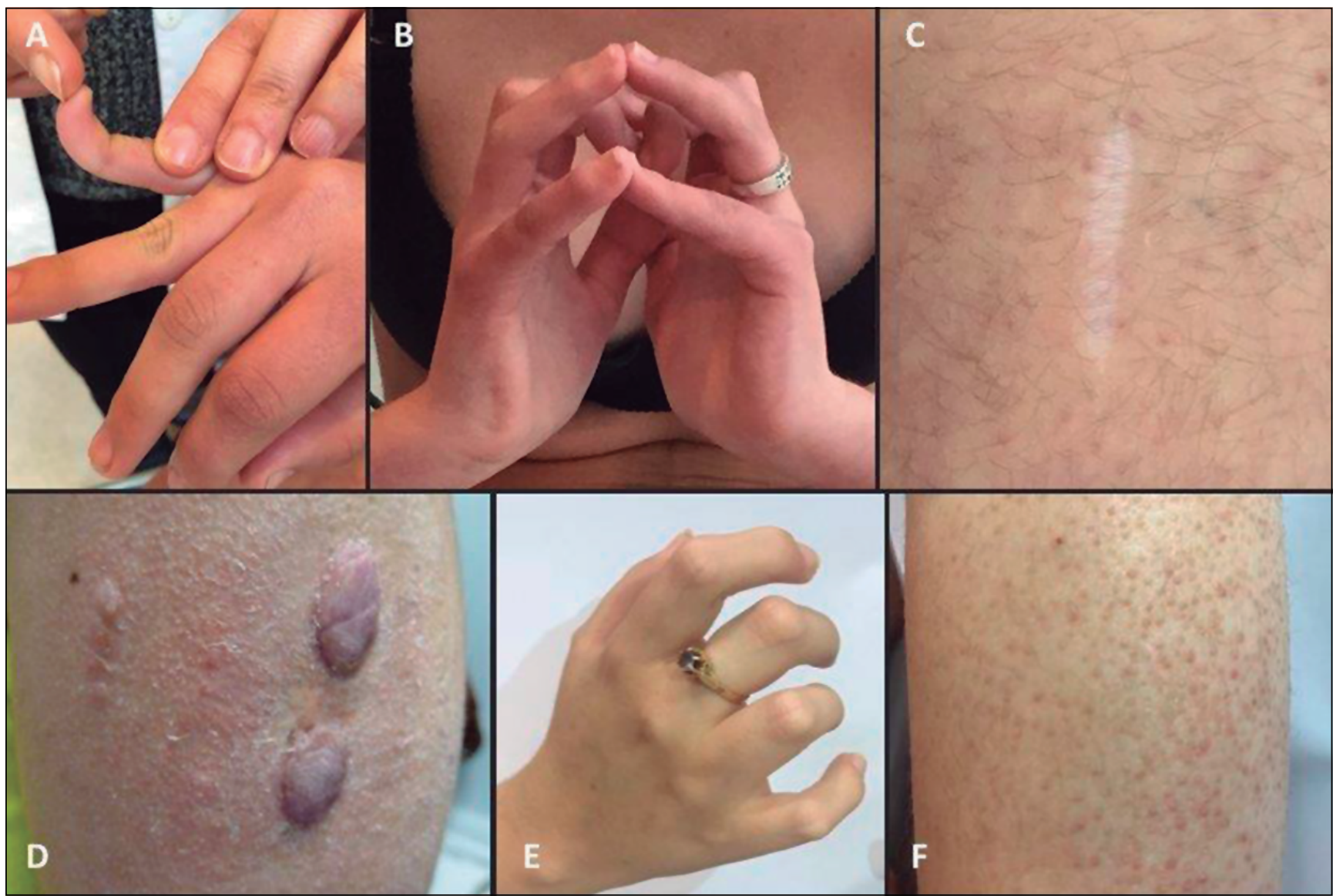

Figura 2. Imágenes A, B y C corresponden a paciente de sexo femenino con diagnóstico de MB. A) Hiperlaxitud articular; B) Contracturas distales de dedos, "signo de Bethlem"; C) Cicatriz en "papel de cigarro". Imágenes D, E y F corresponden a paciente de sexo femenino con DMCU. D) Cicatriz queloide; E) Contracturas distales de dedos; F) Hiperqueratosis pilar. 


\section{Distrofia Muscular Congénita de Ullrich (DMCU)}

El fenotipo clásico ${ }^{16}$, se presenta desde el nacimiento, con hipotonía y debilidad muscular generalizada, asociada en forma característica a una marcada hiperlaxitud de las articulaciones distales de las manos y pies. Pueden coexistir contracturas articulares en codos, rodillas, columna (cifoescoliosis), cuello (tortícolis congénita), además de presentar trastornos en la alimentación, requiriendo de sonda nasogástrica o gastrostomía en los casos más severos ${ }^{17}$. Puede existir debilidad facial, pero los movimientos oculares se encuentran conservados. Los pacientes con fenotipo severo de inicio precoz nunca logran marcha independiente, pudiendo lograr sólo control sedente y desplazamiento por arrastre. Por el contrario, los pacientes con fenotipo moderado, que es el más frecuente, logran la marcha independiente alrededor de los 2 años de vida, con pérdida posterior de habilidades motoras durante la adolescencia o adultez temprana. La debilidad muscular es progresiva, sin embargo son las contracturas de grandes articulaciones, las que más interfieren con la capacidad motora y la deambulación; asociada generalmente a una escoliosis de inicio pre$\mathrm{COZ}$ (etapa escolar) que con mucha frecuencia requiere cirugía $^{18}$. La debilidad de la musculatura respiratoria asociada a escoliosis progresiva favorece el desarrollo de insuficiencia respiratoria de tipo restrictiva. Esta, si bien se desarrolla una vez que se pierde la marcha, puede manifestarse antes con hipoxemia nocturna, que se acompaña de somnolencia diurna y cefalea secundaria a la hipercapnia y disminución progresiva de la capacidad vital forzada. En esta etapa es necesario el apoyo ventilatorio no invasivo (BIPAP) $)^{17}$, lo que debe ser supervisado de manera periódica y precoz.

\section{Miopatías intermedias relacionadas a Colágeno VI}

Grupo de pacientes con M-COLVI que no son fácilmente clasificables como DMCU ni cómo MB. Algunos de estos pacientes han sido clasificados por diversos autores como miopatía relacionada a Colágeno VI, de carácter leve e inicio precoz, puesto que si bien se inician en el período neonatal, logran la marcha independiente que mantienen incluso más allá de la adultez temprana, después de los 20 años. La característica clínica más relevante es el desarrollo de contracturas progresivas desde la niñez, especialmente de codos, tobillos y rodillas, además de escoliosis y espina rígida, pudiendo requerir asistencia y ayudas técnicas para mantener la movilidad. También pueden presentar insuficiencia respiratoria, con disminución en la capacidad vital forzada en la adolescencia temprana ${ }^{13,17}$. Dentro de los reportes de series de casos relacionados con fenotipos intermedios se describe una forma de distrofia muscular de cinturas autosómica dominante ${ }^{19} \mathrm{y}$ una forma de mioesclerosismiopatía autosómica recesiva ${ }^{20}$.

\section{Miopatía de Bethlem (MB)}

La MB es el fenotipo más leve y es extremadamente variable en su presentación clínica. Frecuentemente se presentan otros miembros afectados en la familia junto al caso índice.

Si bien el diagnóstico de MB suele realizarse en adultos, la edad de inicio de los síntomas en la mayoría de los casos es al final de la primera década de la vida, con debilidad leve y algún grado de hiperlaxitud distal, que puede evolucionar posteriormente a contracturas aquileanas, de codos, músculos pectorales, flexores de los dedos y articulaciones interfalángicas, que imposibilitan la extensión de los dedos (ver figura 2B, signo de Bethlem). Destaca también al interrogar en forma dirigida a adultos, la existencia desde la infancia de signos como hipotonía, laxitud articular con luxación de caderas, luxaciones recidivantes de rótula ó malformaciones del pie ${ }^{21}$. La instauración de la enfermedad también puede ser congénita, pero los niños tienen menor compromiso que en DMCU, encontrando deformidad en equinovaro o más frecuentemente, tortícolis y contracturas en dorsiflexión de los pies?. Los pacientes con MB pueden desarrollar escoliosis con espina rígida, que comparada con la DMCU es moderada y requerir ayudas técnicas para deambular por las contracturas ${ }^{21}$. Otra complicación potencial, asociada a la progresión de la enfermedad, es el desarrollo de algún grado de insuficiencia respiratoria de tipo restrictivo, secundario a la combinación de rigidez de la caja torácica con debilidad de la musculatura respiratoria ${ }^{22}$.

\section{Diagnóstico}

La historia clínica dirigida buscando los signos musculoesqueléticos y de piel antes enumerados es fundamental en la sospecha diagnóstica. Estos signos clínicos suelen no asociarse en forma rutinaria a las miopatías, especialmente en aquellos pacientes menos débiles, en quienes los aspectos articulares y esqueléticos predominan por sobre la debilidad. Junto a la anamnesis y el examen físico, los niveles de creatinkinasa séricas y la electromiografía (EMG), siguen jugando un rol importante en la evaluación diagnóstica de un paciente con síndrome miopático. Los niveles de creatinkinasa sanguínea pueden ser normales o estar elevados 2 a 3 veces sobre el valor normal ${ }^{23,24}$ y la EMG corroborará la existencia de un compromiso miopático, descartando a su vez la presencia de compromiso neuropático o de segunda motoneurona.

\section{Biopsia muscular}

El diagnóstico se puede realizar a través de una biopsia muscular; en el caso de la MB, podemos encontrar cambios inespecíficos con variación en el diámetro 
de las fibras, núcleos internalizados y aumento del tejido conectivo endomisial ${ }^{25}$. Por lo anterior es necesario evaluar las anomalías en la expresión del Colágeno VI por medio de técnicas inmunohistoquímicas y, dado que se puede presentar reducción sutil en la marcación, es necesario recurrir a la identificación de otras proteínas de la matriz extracelular como el perlecam (marcación doble) y/o inmunofluorescencia de cultivo de fibroblastos de la piel, para mejorar el rendimiento de la biopsia. Ambos estudios tienen una sensibilidad y especificidad muy alta en el diagnóstico de distrofia muscular de Ullrich (cerca de un 100\%), sin embargo para miopatía de Bethlem la especificidad es menor (63\%). Dado lo anterior, si bien la inmunomarcación de colágeno VI es una herramienta diagnóstica muy útil (especialmente Ullrich), en muchos centros se sigue dando preferencia a la historia clínica e imágenes de resonancia ante sospecha de Bethlem. Por ello, ante la sospecha de Bethlem, el estudio de biopsia muscular se reserva en general para descartar otras afecciones musculares y los estudios de fibroblastos para evaluar el significado del estudio genético ${ }^{26}$.

\section{Estudio genético}

Se han descrito 3 genes principales que codifican el Colágeno VI: COL6A1, COL6A2 y COL6A3, los primeros dos ubicados en el cromosoma 21 (21q22.3), el tercero ubicado en el cromosoma $2(2 q 37,3)^{18}$.

Las M-COLVI pueden ser heredadas de forma autosómica dominante o recesiva. En el caso de la DMCU, generalmente es causada por mutaciones recesivas que llevan a pérdida de función, sin embargo se han descrito algunos casos de mutaciones "de novo" dominantes-negativas. El fenotipo intermedio y la miopatía de Bethlem son mayormente causadas por mutaciones dominantes de severidad variable y menos frecuentemente por mutaciones recesivas 9 . Existe una gran variabilidad fenotípica y de progresión de la enfermedad dentro de una misma familia, etiopatogenia molecular aún desconocida, la cual puede estar relacionada con fenómenos de epigenética, genes modificadores y/o mutaciones del splicing, sin embargo se han reportado también casos de mosaicismos parentales heredados de mutaciones dominantes del colágeno VI que simulan fenómenos de anticipación de la severidad de la enfermedad en generaciones subsecuentes ${ }^{27}$.

La confirmación genética se logra por secuenciación directa de los genes COL6A1, COL6A2 y CO$L 6 A 3$, lo cual puede identificar mutaciones puntuales y deleciones que generan una reducción o alteración de la función del colágeno VI. Las mutaciones más comúnmente encontradas involucran in-frame exón skipping o sustituciones de glicina en el dominio Gky-X-Y motif del colágeno triple helicoidal ${ }^{28}$. No obstante lo anterior, los análisis de secuenciación de los tres genes realizados en $\mathrm{ADN}$ genómico extraído de muestras de sangre periférica, han detectado variantes patogénicas sólo en el $66 \%$ de los pacientes clínicamente clasificados como miopatía de Bethlem clásico y $56 \%$ de los pacientes con miopatía de Bethlem con un fenotipo inusualmente severo, sin embargo para el diagnóstico de DMCU esta cifra es mucho mayor, llegando al diagnóstico hasta un $79 \%$ de los pacientes ${ }^{15}$. La baja tasa de detección encontrada en miopatía de Bethlem se atribuye a la gran heterogeneidad genética de estas afecciones y a que la secuenciación no detecta variantes crípticas o intrónicas profundas.

\section{Resonancia magnética muscular}

La resonancia magnética muscular (RMM) ha surgido como una excelente herramienta de apoyo en el diagnóstico y seguimiento de diversas miopatías y distrofias musculares. Lo anterior se atribuye a diversos factores dentro de los cuales están: su mejor resolución comparado con la tomografía computarizada y ultrasonido, avances en el conocimiento de patrones específicos de compromiso muscular asociados a mutaciones en ciertos genes y la posibilidad de usar scores semicuantitativos para determinar el grado de compromiso muscular, pudiendo ayudar en la elección del mejor músculo para realizar biopsia, objetivar la progresión de la enfermedad y efectos de terapias ${ }^{29}$. En cuanto a los protocolos usados para la adquisición de las imágenes en RMM, existen algunos consensos como el del TREAT-NMD NMR (2009), donde las secuencias T1w son consideradas las más apropiadas para el detalle anatómico y para determinar área o volumen de sección transversal muscular. La secuencia Dixon de tres puntos (PDw MRI) se considera para la cuantificación de la infiltración grasa, siendo una técnica rápida y de utilidad como herramienta pronóstica de la progresión de la enfermedad. La secuencia T2 y sus derivadas permiten evaluar edema, necrosis o inflamación de músculos específicos o de grupos musculares ${ }^{30}$.

Hasta ahora, el análisis del patrón de compromiso muscular se ha realizado principalmente en los miembros inferiores y para una correcta interpretación es ideal una aproximación sistemática en la cual se debe considerar $^{29}$ :

- Distribución de los hallazgos: proximal/distal, anterior/posterior.

- Que músculos se afectan o están selectivamente respetados en cada compartimiento.

- Tipo de compromiso de cada musculo: perifascial, "moteado" o completo.

- Presencia de atrofia, hipertrofia o pseudohipertrofia muscular.

- Estimar el grado de compromiso muscular por infiltración grasa usando un score semi-cuantitativo $^{31,32}$. 
Específicamente, los hallazgos encontrados en la RMM pacientes con M-COLVI han sido ampliamente documentados en la literatura, con un patrón de afectación de las extremidades inferiores característico ${ }^{33,34}$ (Figura 3):

- Se aprecia un compromiso difuso de la musculatura, con infiltración grasa en forma estriada o "tigroide", mayor a proximal; en el caso del muslo mayor en compartimiento anterior y en la pierna se observa un anillo de mayor compromiso entre los músculos gastrocnemios y sóleo.

- Destaca el compromiso selectivo con patrón característico en músculos: a) Vasto lateral, con afección de las fibras periféricas y conservación de las fibras centrales, lo cual le da al músculo un aspecto de "sándwich" 35; b) Recto femoral con una zona anterior de menor compromiso en forma de " $U$ ", llamado "signo del colágeno" "signo del target" o "sombra central"33-35. La sensibilidad y especificidad de estos dos signos es de $90 \%$ y $97,3 \%$ respectivamente ${ }^{35}$.

- Con el uso de la resonancia muscular del cuerpo completo (WB-MRI) se ha caracterizado el compromiso en otros músculos como el tríceps, deltoides, psoas y latísimo del dorso ${ }^{36}$ (Figura 4 y 5).
- Respeto relativo de los músculos sartorio, gracilis y aductor largo ${ }^{31}$.

Si bien este patrón descrito es característico, el aporte de la resonancia para el diagnóstico de las M-COLVI es especialmente relevante en la miopatia de Bethlem, donde tanto el estudio genético como la biopsia muscular no siempre son diagnósticos. Por otro parte, la RMM colabora en el diagnóstico diferencial con las principales patologías clínicamente similares como lo son las miopatías multiminicore, central core, distrofia muscular de Emery-Dreifuss, entre otras miopatías con contracturas y rigidez espinal. Las miopatías central core, frecuentemente asociadas a mutaciones del receptor de la rianodina ( $R Y R 1)$, presentan un patrón característico con compromiso difuso del muslo, que respeta el aductor largo, recto femoral, y gracilis. En la pierna, el sóleo es el músculo más comprometido, seguido por los gastrocnemios y peroneos ${ }^{37,38}$, además de compromiso de la musculatura facial (lengua, músculos temporales, pterigoideos laterales, y maséteros), y musculatura paraespinal ${ }^{36}$. Otras miopatías con cores, como las secundarias a mutaciones de MYH7 y TTN, se caracterizan por ser predominantemente distales, con compromiso del tibial anterior y del grupo peroneo

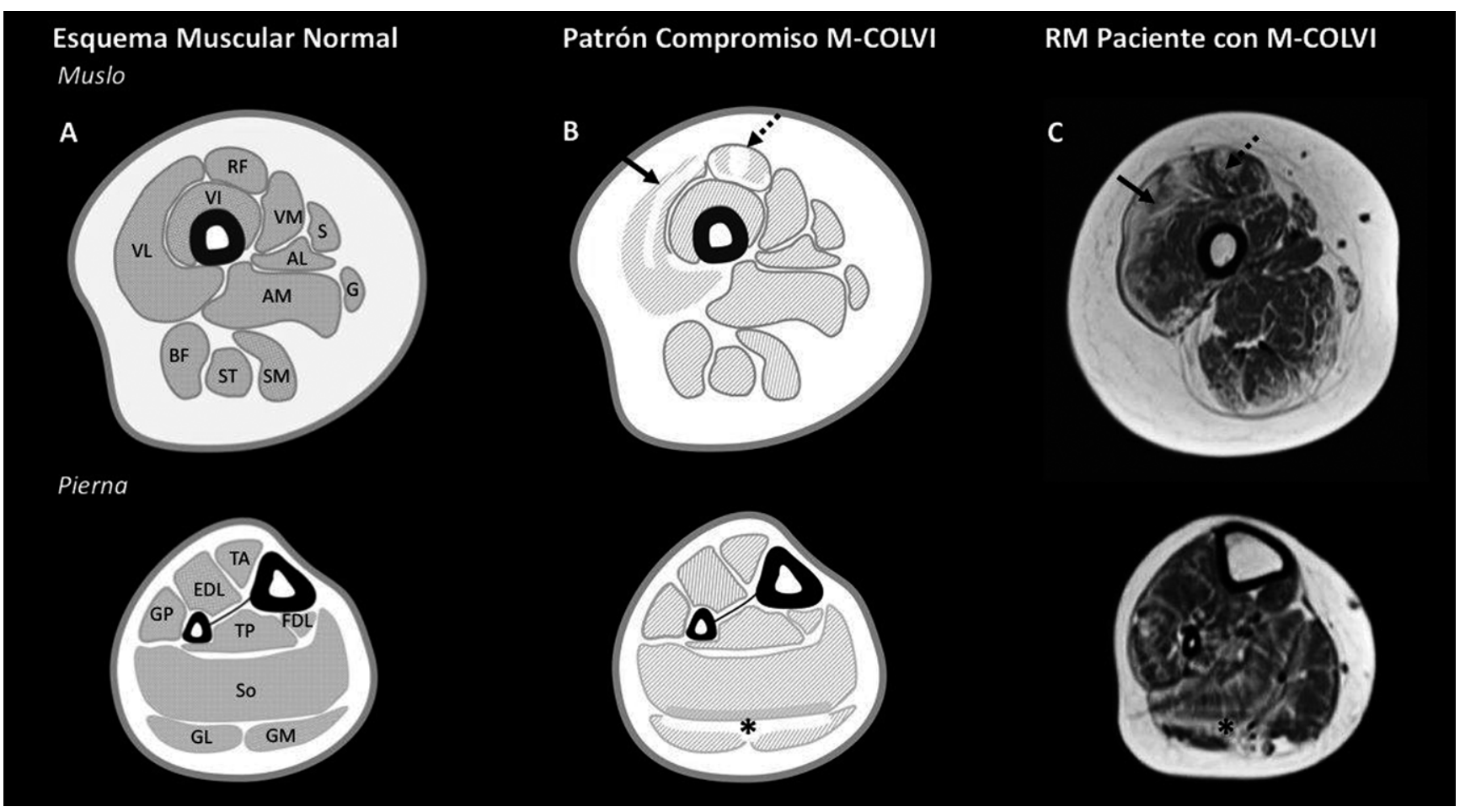

Figura 3. Representación esquemática y comparación con RM muscular en cortes axiales de muslo y pierna. A) Esquema muscular normal: vasto lateral $(\mathrm{VL})$, vasto intermedio $(\mathrm{VI})$, vasto medial $(\mathrm{VM})$, recto femoral (RF), aductor longo (AL), gracilis $(\mathrm{G})$, aductor magno $(A M)$, sartorio $(\mathrm{S})$, semimembranoso (SM), semitendinoso (ST), biceps femoral (BF), tibial anterior (TA), tibial posterior (TP), extensor digital longo (EDL), grupo peroneal $(\mathrm{GP})$, flexor digital longo (FDL), soleo (So), gastrocnemio lateral (GL) y gastrocnemio medial (GM). B) Patrón de compromiso M-COLVI y C) RM de paciente con M-COLVI donde se aprecian hallazgos característicos en el muslo: signo del "sándwich" (flecha continua), signo del "target" (flecha discontinua) y en la pierna, la presencia del "anillo" de infiltración grasa entre el soleo y los gastrocnemios (*). 


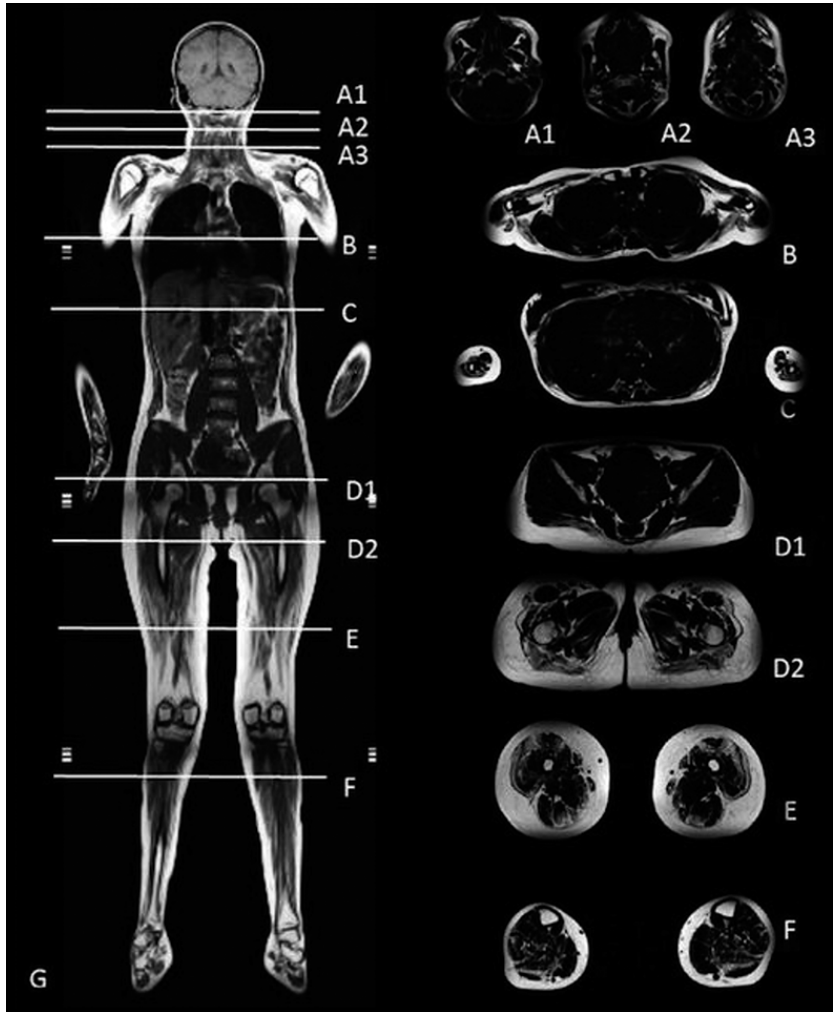

Figura 4. Paciente de 13 años, Miopatía de Bethlem. WBMRI imagen coronal T1 de cuerpo completo e imágenes axiales T1 Dixon grasa segmentarias muestra patrón "atigrado" en diferentes segmentos por alternancia de bandas de hiperintensidad e hipointensidad de señal, especialmente en muslos y piernas. Imágenes axiales A muestran escaso compromiso de musculatura masticatoria. En el tronco (B y C) compromiso graso moderado con mayor intensidad de señal en músculos redondo mayor, serratos y dorsal ancho. En la cintura pélvica (D) compromiso moderado de glúteo mayor, tensor de la fascia lata. En los muslos (E), compromiso moderado de compartimentos anterior y posterior, especialmente vasto lateral, con anillo de menor señal en torno al tendón del recto femoral del cuádriceps. En piernas (F) banda hiperintensa por reemplazo graso de fibras de los músculos sóleo y gastrocnemios

en la primera, y del semitendinoso y peroneo largo, en la segunda ${ }^{39,40}$. Dentro de las miopatías multiminicore, las asociadas a RYR1 y las secundarias a SEPN1 presentan características específicas en RM-M. Ambas comparten un patrón similar al reportado en miopatías central core asociadas a $R Y R 1$, sin embargo la atrofia de los esternocleidomastoideos y de los músculos semimembranosos en SEPN1, permitirían diferenciar a estas dos patología ${ }^{41}$.

\section{Diagnóstico diferencial}

En términos generales, ante pacientes con sospecha de miopatía congénita, hallazgos como presencia de hiperlaxitud articular, CK normal a levemente elevada, ausencia de compromiso cardíaco y una resonancia magnética muscular con patrón característico, pueden diferenciar las M-COLVI de otros síndromes miopáticos.

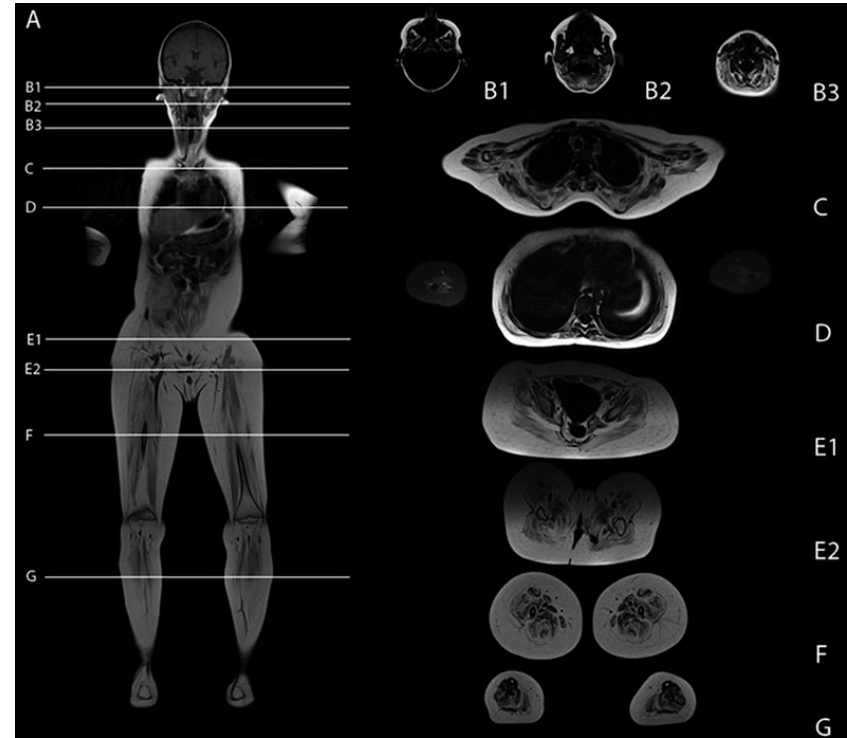

Figura 5. Paciente de 12 años, Distrofia Muscular de Ullrich. WBMRI imagen coronal T1 de cuerpo completo e imágenes axiales T1 Dixon grasa segmentarias. El compromiso es más que moderado en todos los segmentos, manteniéndose el patrón "atigrado" con bandas de mayor y menor señal que se alternan. Se observa menor compromiso de la musculatura masticatoria $(A)$ del aductor largo $(F)$ y los músculos tibiales anterior y posterior (G). Se mantiene anillo de fibras hipointensas, con menor reemplazo graso alrededor del recto femoral del cuádriceps $(\mathrm{F})$ y banda hiperintensa, de mayor reemplazo graso, entre los músculos sóleo y gastrocnemios ( $\mathrm{G})$.

Considerando como característica fundamental la debilidad muscular, las M-COLVI deben diferenciarse de otras miopatías congénitas, que también cursan con hipermovilidad articular y espina rígida como son: 1) las miopatías multiminicore recesivas secundaria a mutacion el gen de la selenoproteina (SEPN1) y gen de la rianodina (RYR1);2) Las miopatías central core secundarias a mutaciones $\mathrm{AR}$ o $\mathrm{AD}$ del $R Y R 1$, las cuales se asocian frecuentemente a luxación congénita de caderas, y otras miopatías con cores secundarias a miosina 7 (MYH7) y titina (TTN). Ante la presencia de contracturas, cuando estas son sutiles, el diagnóstico diferencial principal son las distrofias musculares de cintu$\operatorname{ras}^{19}$ y en presencia de contracturas como rasgo principal, el diagnóstico diferencial es la distrofia muscular de EmeryDreifuss ${ }^{42}$.

Por otro lado, recientemente se han reportado familias con mutaciones del colágeno XII, en quienes se describen fenotipos similares a las M-COLVI, especialmente con fenotipo similar a Bethlem y otras de mayor severidad con presentación desde período neonatal con hipotonía e hiperlaxitud generalizada, sin lograr marcha independiente ${ }^{43,44}$.

Los trastornos del tejido conectivo, sin compromiso miopático primario, como el Síndrome de Ehlers-Danlos $(E D S)^{45}$, el Síndrome de Marfan ${ }^{46}$ y el Síndrome de LoeysDietz ${ }^{47}$, también son diagnósticos diferenciales por la hipermovilidad articular, especialmente en aquellos pacientes en 
quienes la debilidad no es el signo más prominente ${ }^{48,49}$. Particularmente, de los diversos tipos de EDS, aquellos que pueden ser confundidos más fácilmente con miopatías relacionadas a colageno VI son: EDS VIA tipo Cifoescoliótico, VI B Músculo-contractural, VII A Artrocalásico y EDS secundario a déficit de tenascina $\mathrm{X}^{45}$.

\section{Tratamiento}

El manejo inicial consiste en evaluar la extensión de la enfermedad en pacientes con diagnóstico confirmado. En términos generales, se sugiere evaluar el grado de debilidad muscular, compromiso de la movilidad, examinar las articulaciones en busca de contracturas o hiperlaxitud, con evaluación kinésica de contracturas, además de evaluación ortopédica o necesidad de cirugía en caso de contractura del talón de Aquiles y evaluación de la función respiratoria y mecánica ventilatoria. En pacientes con UCMD se sugiere además evaluar la presencia de escoliosis, displasia del desarrollo de la cadera, y trastornos de deglución ${ }^{11,15}$.

Luego de evaluar la extensión del compromiso, el foco del manejo está en el apoyo médico general y en la prevención y tratamiento de las complicaciones que desarrollan estos pacientes. El manejo respiratorio por sí solo ha aumentado la sobrevida de los pacientes con $\mathrm{UCMD}^{9}$. El soporte ventilatorio nocturno es requerido para manejar la hipoventilación nocturna que se produce usualmente en las primeras décadas de la vida de pacientes con UCMD. Pacientes con fenotipos más severos pueden desarrollar un disfunción diafragmática, por lo que su función también debiese ser evaluada ${ }^{50}$. Todos los pacientes requieren manejo kinésico activo para promover la movilidad e independencia funcional. En pacientes con UCMD la inmovilización axial temprana es importante para promover la postura y proteger contra el desarrollo de escoliosis. La escoliosis en pacientes con UCMD se desarrolla frecuentemente en la primera o segunda década y usualmente requiere manejo activo incluyendo cirugía en muchos casos. En pacientes con BM el manejo de las contracturas se logra usualmente con fisioterapia y ortopedia, mientras que los pacientes con UCMD estas son más agresivas y pueden requerir cirugía. Se sugiere realizar profilaxis mediante vacunación y utilización temprana de antibióticos para prevenir infecciones respiratorias en ambos trastornos ${ }^{15}$.

\section{Conclusión}

Las M-COLVI están dentro de las miopatías congénitas más frecuentes, necesitándose para su diagnóstico un alto índice de sospecha, donde la historia clínica y hallazgos al exámen físico son orientadores. Se deben tener en cuenta frente a pacientes de cualquier edad, con antecedentes de hipotonía y/o retraso del desarrollo psicomotor, desde leves a severos, en quienes la debilidad no es necesariamente la característica más prominente, sino el hallazgo de hiperlaxitud y/o contracturas articulares, asociado a compromiso de la piel característico y ausencia de compromiso cardiovascular. Tanto la necesidad de realizar consejo genético y prevenir complicaciones, como los avances de terapias en desarrollo, hacen necesario llegar a un diagnóstico específico de estas condiciones. Los métodos diagnósticos tradicionales como la biopsia muscular, estudios genéticos de última generación y la resonancia muscular son de gran aporte, sin embargo, el proceso diagnóstico puede variar según la disponibilidad de estas herramientas y la experiencia de cada centro clínico (figura 6). La resonancia muscular es una herramienta que aporta en el diagnóstico y que debe ser considerada especialmente ante la sospecha de M-COLVI tipo Bethlem, en el diagnóstico diferencial con otras mio-

Figura 6. Resumen M-COLVI.

\section{Miopatías Relacionadas a Colágeno VI}

\section{¿Cuándo Sospecharlas?}

En todo niño con retraso del desarrollo motor o limitación funcional por debilidad de la musculatura esquelética y contracturas articulares, que adicionalmente presente una o más de las siguientes caracteristicas:

- Algún familiar con diagnóstico de M-COLVI. Hiperlaxitud de las articulaciones distales de manos y pies. Antecedentes de luxación de cadera, deformidad de otras Antecedentes de luxación de ca

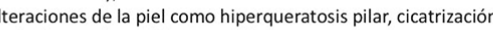
queloide o en "papel de cigarro".

¿Como Identificarlas?

Anamnesis y examen físico completos. Niveles normales o leve elevación de CK.

Electromiografía con reporte de patrón miopático.

Otras pruebas diagnósticas como biopsia muscular y estudio

genético, se aconseja sean dirigidas por un especialista en enfermedades neuromusculares.
Aporte de la Resonancia Magnética Muscular

- Diagnóstico de M-COLVI por patrones imagenologicos especificos y su correlacion con mutaciones en genes COL6A1, COL6A2 y COL6A3.

Permite realizar diagnósticos diferenciales con otros sindromes miopáticos.

Herramienta util en la elección de musculos optimos para la realizacion de la biopsia.

Seguimiento de la evolucion de pacientes con M-COLVI por la posibilidad de usar scores semi-cuantitativos para determinar el grado de compromiso muscular. 
patías, en la interpretación de resultados de estudios genéticos y de biopsia, además de objetivar mejorías con los tratamientos.

\section{Conflicto de intereses}

Los autores declaran no tener conflicto de intereses.

\section{Referencias}

1. Kaplan J-C. GeneTable of Neuromuscular Disorders [Internet]. World Muscle Society. 2017 [cited 2017 Oct 26]. Available from: http://www. musclegenetable.fr

2. Schorling D, Kirschner J, Bönnemann C. Congenital Muscular Dystrophies and Myopathies: An Overview and Update. Neuropediatrics [Internet]. 2017;48(4):247-61. Available from: http://www.ncbi.nlm.nih.gov/ pubmed/28669131\%0Ahttp:// www.thieme-connect.de/DOI/ DOI?10.1055/s-0037-1604154

3. Silvestri NJ, Ismail H, Zimetbaum P, Raynor EM. Cardiac involvement in muscular dystrophies. Muscle Nerve [Internet]. 2017; (Accepted Author Manuscript.). Available from: http:// dx.doi.org/10.1002/mus.26014

4. Amburgey K, McNamara N, Bennett LR, McCormick ME, Acsadi G, Dowling JJ. Prevalence of congenital myopathies in a representative pediatric united states population. Ann Neurol. 2011;70(4):6625.

5. Darin N, Kimber E, Kroksmark AK, Tulinius M. Multiple congenital contractures: Birth prevalence, etiology, and outcome. J Pediatr. 2002;140(1):61-7.

6. Norwood FLM, Harling C, Chinnery PF, Eagle M, Bushby K, Straub V. Prevalence of genetic muscle disease in Northern England: in-depth analysis of a muscle clinic population. Brain [Internet]. 2009;132(Pt 11):3175-86. Available from: http://www.pubmedcentral.nih.gov/ articlerender.fcgi? artid $=4038491 \&$ tool $=p$ mcentrez\&rendertype $=$ abstract

7. Díaz JJ, Suazo RL, Castiglioni TC, Bevilacqua RJA. Utilidad de la resonancia magnética en el diagnóstico de las enfermedades musculares hereditarias. Rev Chil Radiol [Internet]. 2015;21(4):144-50. Available from: http://www.scielo.cl/scielo. php?script=sci_arttext\&pid=S071793082015000400005\&lng $=$ en\&nrm $=$ iso\&t lng $=$ en

8. Conti Reed U, Gobbo Ferreira L, Liu EC, et al. Ullrich congenital muscular dystrophy and bethlem myopathy: Clinical and genetic heterogeneity. Arq Neuropsiquiatr. 2005;63(3 B): 785-90.

9. Bonnemann CG. The collagen VI-related myopathies: Muscle meets its matrix. Nature Reviews Neurology. 2011. p. 37990.

10. Tagliavini F, Pellegrini C, Sardone F, et al. Defective collagen VI $\alpha 6$ chain expression in the skeletal muscle of patients with collagen VI-related myopathies. Biochim Biophys Acta - Mol Basis Dis. 2014;1842(9):1604-12.

11. Bushby KMD, Collins J, Hicks D. Collagen type VI myopathies. Adv Exp Med Biol. 2014;802:185-99.

12. Maraldi NM, Sabatelli P, Merlini L, et al. Collagen VI myopathies: pathogenic mechanism and therapeutic strategies. Ital J Anat Embryol Vol 116, No 12011 [Internet]. 2011; Available from: http:// fupress.net/index.php/ijae/article/ view/10121/9359.

13. Bönnemann CG. The collagen VI-related myopathies. Ullrich congenital muscular dystrophy and Bethlem myopathy. Handbook of Clinical Neurology. 2011. 81-96 p.

14. Sabatelli P, Gara SK, Grumati P, et al. Expression of the collagen VI $\alpha 5$ and $\alpha 6$ Chains in normal human skin and in skin of patients with collagen VIrelated myopathies. J Invest Dermatol. 2011;131(1):99-107.

15. Lampe AK, Flanagan KM, Bushby KM, Hicks D. Collagen Type VI-Related Disorders [Internet]. GeneReviews. 2012 [cited 2017 Nov 26]. p. 1-18. Available from: https://www.ncbi.nlm.nih.gov/ books/NBK1503/

16. Camacho Vanegas O, Bertini E, Zhang RZ, et al. Ullrich scleroatonic muscular dystrophy is caused by recessive mutations in collagen type VI. Proc Natl Acad Sci USA [Internet]. 2001;98(13):7516-21. Available from: http://www.pubmedcentral.nih.gov/ articlerender.fcgi?artid $=34700 \&$ tool $=\mathrm{pmc}$ entrez\&rendertype $=$ abstract.

17. Nadeau A, Kinali M, Main M, et al. Natural history of Ullrich congenital muscular dystrophy. Neurology. 2009;73(1):25-31.

18. Briñas L, Richard $P$, Quijano-Roy S, et al. Early onset collagen VI myopathies: Genetic and clinical correlations. Ann Neurol. 2010;68(4):511-20.

19. Scacheri PC, Gillanders EM, Subramony $\mathrm{SH}$, et al. Novel mutations in collagen VI genes: expansion of the Bethlem myopathy phenotype. Neurology [Internet]. 2002;58(4):593-602. Available from: http://www.ncbi.nlm.nih.gov/ pubmed/11865138

20. Merlini L, Martoni E, Grumati P, et al. Autosomal recessive myosclerosis myopathy is a collagen VI disorder. Neurology. 2008;71(16):1245-53.

21. Jöbsis GJ, Boers JM, Barth PG, De Visser M. Bethlem myopathy: A slowly progressive congenital muscular dystrophy with contractures. Brain. 1999;122(4):649-55.

22. Haq RU, Speer MC, Chu ML, Tandan R. Respiratory muscle involvement in Bethlem myopathy. Neurology. 1999;52(1):174-6.

23. Sparks SE, Quijano-Roy S, Harper A, et al. Congenital Muscular Dystrophy Overview [Internet]. GeneReviews $\left.{ }^{\circledR}\right)$. 2012 [cited 2017 Nov 29]. Available from: https://www.ncbi.nlm.nih.gov/books/ NBK1291/

24. Lee JH, Shin HY, Park HJ, Kim SH, Kim SM, Choi Y-C. Clinical, Pathologic, and Genetic Features of Collagen VI-Related Myopathy in Korea. J Clin Neurol [Internet]. 2017;13(4):331-9. Available from: https://www.thejcn.com

25. Deconinck N, Richard P, Allamand V, et al. Bethlem myopathy: long-term follow-up identifies COL6 mutations predicting severe clinical evolution. J Neurol Neurosurg Psychiatry [Internet]. 2015;86(12):1337-46. Available from: http://jnnp.bmj.com/cgi/doi/10.1136/ jnnp-2013-307245.

26. Allamand V, Merlini L, Bushby K. 166th ENMC International Workshop on Collagen type VI-related Myopathies, 2224 May 2009, Naarden, The Netherlands. Neuromuscul Disord. 2010;20(5):346-54.

27. Donkervoort $\mathrm{S}, \mathrm{Hu} \mathrm{Y}$, Stojkovic T, et al. Mosaicism for dominant collagen 6 mutations as a cause for intrafamilial phenotypic variability. Hum Mutat. 2015;36(1):48-56.

28. Butterfield RJ, Foley AR, Dastgir J, et al. Position of glycine substitutions in the triple helix of COL6A1, COL6A2, and COL6A3 is correlated with severity and mode of inheritance in collagen vi myopathies. Hum Mutat. 2013;34(11):1558-67.

29. Cejas CP, Serra MM, Galvez DFG, et al. Muscle MRI in pediatrics: clinical, pathological and genetic correlation. Pediatr Radiol. 2017;47(6):724-35.

30. Hollingsworth KG, de Sousa PL, Straub V, Carlier PG. Towards harmonization 
of protocols for MRI outcome measures in skeletal muscle studies: Consensus recommendations from two TREATNMD NMR workshops, 2 May 2010, Stockholm, Sweden, 1-2 October 2009, Paris, France. Neuromuscul Disord. 2012;22(SUPPL. 2).

31. Mercuri E, Pichiecchio A, Allsop J, Messina S, Pane M, Muntoni F. Muscle MRI in inherited neuromuscular disorders: Past, present, and future. Journal of Magnetic Resonance Imaging. 2007. p. 433-40.

32. Mercuri E, Jungbluth H, Muntoni F. Muscle imaging in clinical practice: diagnostic value of muscle magnetic resonance imaging in inherited neuromuscular disorders. Curr Opin Neurol [Internet]. 2005;18(5):526. Available from: papers://4b497daf11fc-4b77-bfc5-b5ecbecc5200/Paper/ p290\%5Cnhttp://www.ncbi.nlm.nih.gov/ pubmed/16155435

33. Mercuri E, Lampe A, Allsop J, et al. Muscle MRI in Ullrich congenital muscular dystrophy and Bethlem myopathy. Neuromuscul Disord. 2005;15(4):303-10.

34. Morrow JM, Pitceathly RDS, Quinlivan RM, Yousry TA. Muscle MRI in Bethlem myopathy. BMJ Case Rep [Internet]. 2013;2013:1-2. Available from: http:// casereports.bmj.com/cgi/doi/10.1136/bcr2013-008596

35. Fu J, Zheng YM, Jin SQ, et al. "Target” and "sandwich" signs in thigh muscles have high diagnostic values for collagen
VI-related myopathies. Chin Med J (Engl). 2016;129(15):1811-6.

36. Quijano-Roy S, Avila-Smirnow D, Carlier RY, et al. Whole body muscle MRI protocol: Pattern recognition in early onset NM disorders. Neuromuscul Disord. 2012;22(SUPPL. 2).

37. Straub V, Carlier PG, Mercuri E. TREATNMD workshop: Pattern recognition in genetic muscle diseases using muscle MRI. 25-26 February 2011, Rome, Italy. Neuromuscul Disord. 2012;22(SUPPL. 2).

38. Jungbluth H, Davis MR, Müller C, et al. Magnetic resonance imaging of muscle in congenital myopathies associated with RYR1 mutations. Neuromuscul Disord. 2004;14(12):785-90.

39. Quijano-Roy S, Carlier RY, Fischer D. Muscle Imaging in Congenital Myopathies. Semin Pediatr Neurol. 2011;18(4):221-9.

40. Pfeffer G, Elliott HR, Griffin H, et al. Titin mutation segregates with hereditary myopathy with early respiratory failure. Brain. 2012;135(6):1695-713.

41. Hankiewicz K, Carlier RY, Lazaro L, et al. Whole-body muscle magnetic resonance imaging in SEPN1-related myopathy shows a homogeneous and recognizable pattern. Muscle and Nerve. 2015;52(5):728-35.

42. Pepe G, Bertini E, Bonaldo P, et al. Bethlem myopathy (BETHLEM) and Ullrich scleroatonic muscular dystrophy: 100th ENMC International Workshop, 23-24 November 2001, Naarden, NLD. Neuromuscular Disorders. 2002. p. 984-
93.

43. Hicks D, Farsani GT, Laval S, et al. Mutations in the collagen XII gene define a new form of extracellular matrixrelated myopathy. Hum Mol Genet. 2014;23(9):2353-63.

44. Punetha J, Kesari A, Hoffman EP, et al. Novel Col12A1 variant expands the clinical picture of congenital myopathies with extracellular matrix defects. Muscle and Nerve. 2017;55(2):277-81.

45. Syx D, De Wandele I, Rombaut L, Malfait F. Hypermobility, the Ehlers-Danlos syndromes and chronic pain. Clin Exp Rheumatol. 2017;35(5):S116-22.

46. Dietz HC. Marfan Syndrome [Internet]. GeneReviews $\left.{ }^{\circledR}\right) .2017$ [cited 2017 Dec 1]. Available from: https://www.ncbi.nlm. nih.gov/books/NBK1335/

47. MacCarrick G, Black JH, Bowdin S, et al. Loeys-Dietz syndrome: A primer for diagnosis and management. Genet Med. 2014;16(8):576-87.

48. Voermans NC, Bönnemann CG, Huijing PA, et al. Clinical and molecular overlap between myopathies and inherited connective tissue diseases. Neuromuscular Disorders. 2008. p. 843-56.

49. Donkervoort S, Bonnemann CG, Loeys B, Jungbluth H, Voermans NC. The neuromuscular differential diagnosis of joint hypermobility. Am J Med Genet Part C Semin Med Genet. 2015;169(1):23-42.

50. Quijano-Roy S, Khirani S, Colella M, et al. Diaphragmatic dysfunction in Collagen VI myopathies. Neuromuscul Disord. 2014;24(2):125-33. 